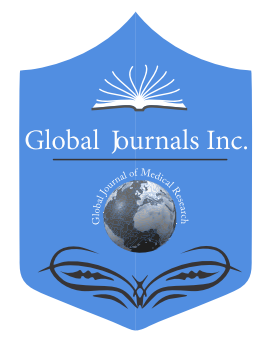

GLOBAL JOURNAL OF MEDiCAL RESEARCH: K

INTERDISCIPLINARY

Volume 21 Issue 7 Version 1.0 Year 2021

Type: Double Blind Peer Reviewed International Research Journal

Publisher: Global Journals

Online ISSN: 2249-4618 \& Print ISSN: 0975-5888

\title{
Outcome Assessment in Case of Severe COVID-19 Patients Treated with Remdesivir
}

By Dr. Shailendranath Biswas, Dr. S K Ballav, Dr. Sheikh Farid Uddin Ahmed, Dr. Utpal Kumar Chanda, Dr. Tahmida Khanom, Dr. Mohaiminul Haque, Dr. Ripon Roy \& Dr. Avijit Kumar Mridha

Khulna Medical College Hospital

Abstract-Background: A sudden outbreak of a novel coronavirus disease (covid-19) pandemic has thrown challenges in searching out a truly effective drug or vaccine to minimize the heavy toll of mortality and morbidity worldwide. But still, now humanity is lagging in finding such an agent that can be labelled as absolutely efficacious.

Methods: We conducted a prospective observational cohorttrial of injectable Remdesivir in the case of hospitalized patients presenting with features of respiratory tract infection and diagnosed as COVID-19 pneumonia by RT-PCR for COVID-19 test and categorized as severe COVID-19 cases as per national guidelines criteria. Patients were treated with injectable Remdesivir (200mg on day 1 , followed by $100 \mathrm{mg}$ daily for up to 05 additional days) along with other standard treatment protocols. The primary outcome of the study was the time to recovery, defined by improvement in clinical and laboratory parameters whether discharge from the hospital or not (hospitalization for infection-control purposes only).

Keywords: SARS-CoV-2, oximetry, mechanical ventilation, radiological improvement.

GJMR-K Classification: NLMC Code: WB 115

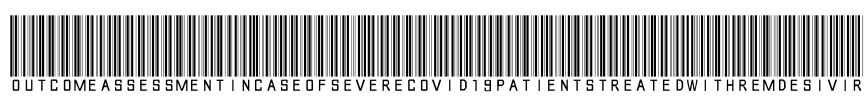

Strictly as per the compliance and regulations of:

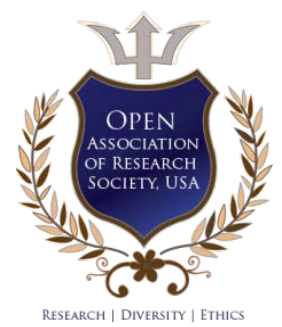

(c) 2021. Dr. Shailendranath Biswas, Dr. S K Ballav, Dr. Sheikh Farid Uddin Ahmed, Dr. Utpal Kumar Chanda, Dr. Tahmida Khanom, Dr. Mohaiminul Haque, Dr. Ripon Roy \& Dr. Avijit Kumar Mridha. This research/review article is distributed under the terms of the Attribution-NonCommercial-NoDerivatives 4.0 International (CC BY-NC-ND 4.0). You must give appropriate credit to authors and reference this article if parts of the article are reproduced in any manner. Applicable licensing terms are at https://creativecommons.org/licenses/by-nc-nd/4.0/. 


\title{
Outcome Assessment in Case of Severe COVID-19 Patients Treated with Remdesivir
}

\author{
Dr. Shailendranath Biswas ${ }^{\alpha}$, Dr. S K Ballav ${ }^{\sigma}$, Dr. Sheikh Farid Uddin Ahmed ${ }^{\circ}$, Dr. Utpal Kumar Chanda ${ }^{\omega}$, \\ Dr. Tahmida Khanom ${ }^{*}$, Dr. Mohaiminul Haque ${ }^{\S}$, Dr. Ripon Roy ^ \& Dr. Avijit Kumar Mridha ${ }^{\vee}$
}

\begin{abstract}
Background: A sudden outbreakof a novel coronavirus disease (covid-19) pandemic has thrown challenges in searching out a truly effective drug or vaccine to minimize the heavy toll of mortality and morbidity worldwide. But still, now humanity is lagging in finding such an agent that can be labelled as absolutely efficacious.
\end{abstract}

Methods: We conducted a prospective observational cohorttrial of injectable Remdesivir in the case of hospitalized patients presenting with features of respiratory tract infection and diagnosed as COVID-19 pneumonia by RT-PCR for COVID-19 test and categorized as severe COVID-19 cases as per national guidelines criteria. Patients were treated with injectable Remdesivir (200mg on day 1, followed by $100 \mathrm{mg}$ daily for up to 05 additional days) along with other standard treatment protocols. The primary outcome of the study was the time to recovery, defined by improvement in clinical and laboratory parameters whether discharge from the hospital or not (hospitalization for infection-control purposes only).

Results: We enrolled a total of 53 patients in this study who fulfilled the inclusion and exclusion criteria. After getting treatment with Remdesivir, the participants had a median recovery time of 10 days). The in-hospital mortality was $14 \%$ by day 15 . Serious adverse events were reported in the case $16 \%$ of patients in the form of renal impairment $(08 \%)$ and drug rash (08\%).

Conclusion: The results obtained from our study show that Remdesivir can play a significant role in shortening hospital stay, hastening clinical recovery, and reducing serious complications in hospitalized patients suffering from severe COVID-19 disease.

Keywords: SARS-CoV-2, oximetry, mechanical ventilation, radiological improvement.

Corresponding Author a: Junior Consultant, Department of Medicine, Khulna Medical College Hospital, Khulna, Bangladesh.

e-mail: drshailendranath12@gmail.com

Author o: Professor, Ex Principle and Head of the Department ENT, Khulna Medical College Hospital, Khulna, Bangladesh.

Author p: Associate Professor, Department of Anaesthesiology, Khulna Medical College Hospital, Khulna, Bangladesh.

Author w: Assistant Professor, Department of Medicine, Khulna Medical College Hospital, Khulna, Bangladesh.

Author ¥: Indoor Medical Officer (IMO), Khulna Medical College Hospital, Khulna, Bangladesh.

Author \&: Medical Officer, Khulna Medical College Hospital, Khulna, Bangladesh.

Author x: Assistant Registrar, Khulna Medical College Hospital, Khulna, Bangladesh.

Author v: Medical Officer, Khulna Medical College Hospital, Khulna, Bangladesh.

\section{INTRODUCTION}

I he coronavirus disease-2019 pandemic popularly known as COVID-19is an infectious disease caused by severe acute respiratory syndrome coronavirus-2 (SARS-CoV-2), a novel coronavirus [1]. Since its origin, in December 2019 in Hubei Province of China, the novel coronavirus has devastated lives and livelihoods worldwide[2].Though it's not new for this earth to fight pandemics, this time it has challenged us to rethink our global heath achievements and brought about a major socioeconomic breakdown around the world. Moreover, the pandemic has been associated with a mortality rate of all times (10\%) [3].

Coronaviruses can cause a wide range of respiratory infections in human hosts. SARS-CoV-2 is a positive-sense single-stranded ribonucleic acid (RNA) virus with an incubation period of up to 14 days and an infectivity rate $(\mathrm{R} 0)$ from 1.5 to more than 6 in some areas of the world[4]. Many infected patients are asymptomatic and about $80 \%-90 \%$ have mild or moderate disease[5]. Currently, there is no antiviral drug to be claimed as absolutely beneficial and vaccines have got approval just recently. So, the search for an antiviral is still going on.

Remdesivir is a prodrug of a nucleotide analogue that is intracellularly metabolized to an analogue of adenosine triphosphate that inhibits viral RNA polymerases [6]. Remdesivir has broad-spectrum activity against members of several virus families, including filoviruses (e.g., Ebola) and coronaviruses (e.g., SARS-CoV and Middle East respiratory syndrome coronavirus [MERS-CoV]) and has shown prophylactic and therapeutic efficacy in nonclinical models of these coronaviruses[7], [8]. In vitro testing has also shown that remdesivir has activity against SARS-CoV-2[9]. Besides, in nonhuman primate studies, remdesivir initiated 12 hours after inoculation with MERS-CoV ${ }^{10,11}$ reduced lung virus levels and lung damage[10]. Remdesivir appears to have a favorable clinical safety profile, as reported based on experience in approximately 500 persons, including healthy volunteers and patients treated for acute Ebola virus infection and supported by data (on file and shared with the World Health Organization [WHO]) [11], [12]. In this report, we describe outcomes in a cohort of patients hospitalized for severe Covid-19 
who were treated with remdesivir on a compassionateuse basis.

So, as a part of the intensive search for an effective antiviral agent, we designed a randomized double-blind trial of Remdesivir on hospitalized severe COVID-19 patients (after laboratory confirmation). Based on initial research and with the approval of the ethical committee we conducted the study on patients in Corona dedicated Hospital, Khulna; Flu corner and Isolation Ward of Khulna Medical College Hospital and Gazi Medical College Hospital.

\section{Methods}

\section{a) Design}

The enrolment for the above-designed study started on August 27, 2020, and ended on October 20, 2020. We conducted the trial simultaneously in three institutes under two different authorities namely Corona Dedicated hospital, Khulna and Isolation and Flu Corner of Khulna Medical College Hospital (Government Institutes), and Gazi Medical College Hospital (Private Institute). After strict maintenance of the inclusion and exclusion criteria patients were selected for this interventional trial. At enrolment, we followed the national guideline of Bangladesh for COVID-19 management published by DGHS (Directorate General of Health Services), Bangladesh to categorize the patients according to disease severity. We allocated patients with severe COVID-19 infections for the study as per the research protocol. Severe cases were defined as cases having either respiratory distress ( $\geqq 30$ breaths/ min); or finger oxygen saturation $\leq 93 \%$ at rest, or arterial partial pressure of oxygen ( $\mathrm{PaO} 2) /$ fraction of inspired oxygen $\quad(\mathrm{FiO} 2) \leqq 300 \mathrm{mmHg} \quad(1 \mathrm{mmHg}=0.133 \mathrm{kPa})[13]$. The trial was designed to use injectable Remdesivir in the dose of 200-mg on day 1, followed by $100 \mathrm{mg}$ daily on days 2 to5 or until hospital discharge or death. All the enrolled patients had simultaneously other supportive care according to the standard treatment protocol practiced throughout the country as per national guidelines. Any other experimental treatment or alternative medicines (widely practiced in the country) or any OTC drug or use of any other medications designated as a specific treatment for Covid-19 were restricted throughout the study period (whether such medications could have been started before enrolment in this trial or not).

We took approval by the Ethical Clearance Committee of both Khulna Medical College (for Corona Dedicated hospital, Khulna and Isolation and Flu Corner of Khulna Medical College Hospital) and Gazi Medical College for conducting the trial. The study was also oversaw by an independent data and safety monitoring board from time to time. Informed written consent was obtained from each patient or from their legal guardian in case the patient was unable to provide consent.

\section{b) Procedures}

There were daily routine follow-ups of the patients in some pre-fixed clinical parameters. Both subjective and objective assessments were included in these regular check-ups. Thorough physical examination with special attention to general and cardiorespiratory systems was a routine task. All routine and special investigations and any investigation felt necessary during hospitalization were done from time to time. Any reported or observed adverse events were recorded and any correlation either with an increase in severity from day 1 or suspected drug-related hypersensitivity reactions was searched.

\section{c) Outcomes}

The primary outcome of this study was the time to recovery. According to the national guideline, this recovery was defined as the first day, during the 14 days after enrolment, on which a patient met the clinical criteria for recovery like a resolution of fever without the use of fever-reducing medications e.g paracetamol for at least 3 (three) days and significant improvement in the respiratory symptoms (e.g., cough, shortness of breath) for 3 days [13].

There were several secondary outcomes of the study. Among them, the key secondary outcome was mortality from the date of enrolment until 14 days later. Other secondary outcomes included the time to improvement in oxygen saturation $(\mathrm{SpO} 2)$ by pulse oximetry upto day 14; the incidence of new mechanical ventilation use within 14 days from the day of enrolment; duration of hospitalization from the day of randomization until the date of hospital discharge or date of death from any cause, whichever came first, assessed up to 14days and cumulative incidence of serious adverse event assessed on a routine basis from day 1 of enrolment to 14th day and radiological improvement after intervention.

\section{d) Statistical Analysis}

The primary analysis was a stratified log-rank test of time to recovery with Remdesivir with stratification by disease severity (the actual severity at baseline). For the analysis of time to-recovery and time-toimprovement outcomes, data for patients who did not recover and data for patients who died were censored at day 14.

Patients were subgrouped in these study according to several predetermined criteria like age (18 to 39 years, 40 to 64 years, o 265 years), sex, race, socio-economic condition, disease severity at enrolment (according to stratification criteria), duration of symptoms before hospitalization, and presence of coexisting conditions. (See the protocol for more information about the trial methods.) For the assessment of the effect of disease severity on treatment benefit (recovery and mortality), post hoc 
analyses evaluated interactions of efficacy with baseline clinical data (as a continuous variable).

\section{Results}

A total of 67 patients were assessed for eligibility. Among them, 53 fulfilled all the inclusion and exclusion criteria. But there was discontinuation in the study due to withdrawal of consent in the case of 03 patients. So, 50 patients continued the trial and all of them completed the study through 14 days, recovered, or died.

The minimum age of presentation was 31 years and the maximum was 87 years. The mean age of the patients was 57.46 years. Among the patient's majority, 23 (46\%) belonged to 51-60 years of age followed by $11(22 \%)$ in the $61-70$ years group and $09(18 \%)$ in the 41-50 years aged group (Figure 1 ).

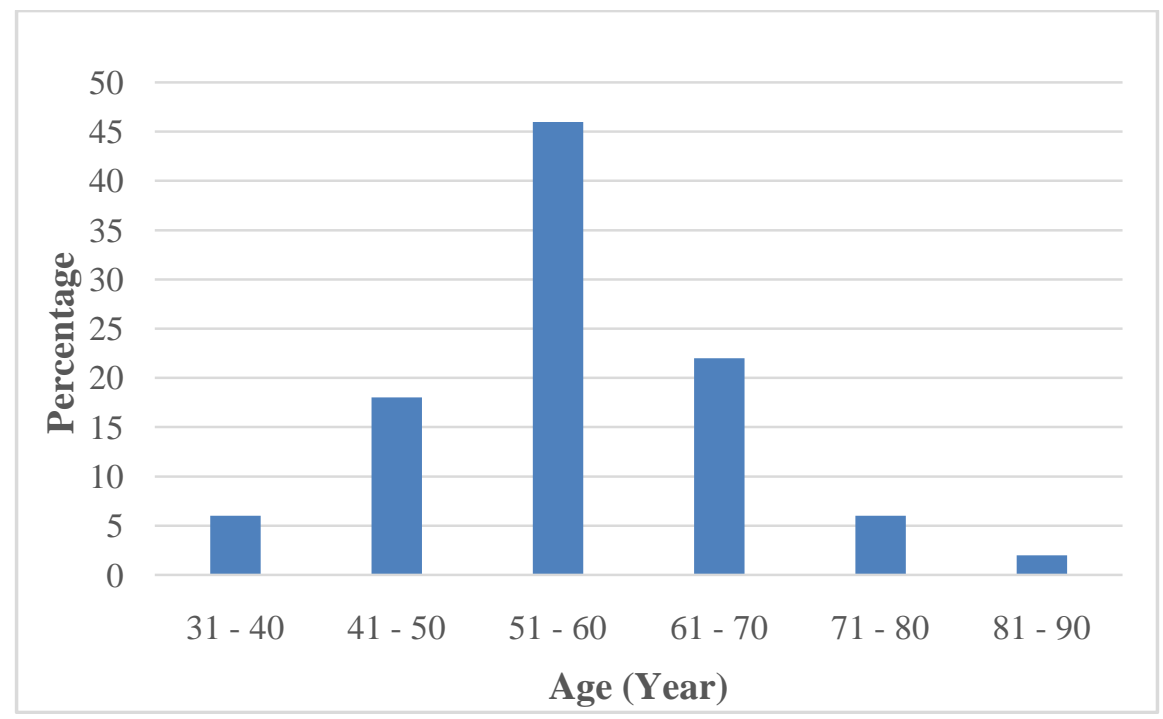

Figure 1: Age Distribution of the Patients

Of the participants $44(88 \%)$ were male and 06(12\%) were female. Among 50 patients $41(82 \%)$ were married and the remaining 09(18\%) were widowed. Occupational analysis of the patients showed that most of them 16(32\%) were business persons and 15(30\%) were service-holders, and the remaining $13(26 \%)$ had other occupations while 06(12\%) females were housewives. If we focus on their socio-economic status, we find that $21(42 \%)$ patients belonged to the upper- middle-class followed by $20(40 \%)$ from the lower middle class, $07(14 \%)$ from the upper class, and the rest of them from the lower class (02, 04\%). In this study majority of the patients had graduation or higher education 25 (50\%) where others had studied either upto primary school $\left(5^{\text {th }}\right.$ grade) $(10,20 \%)$ or, secondary school $\left(10^{\text {th }}\right.$ grade) $(08,16 \%)$, or Higher secondary $\left(12^{\text {th }}\right.$ grade) $(10,20 \%)$ and $07(14 \%) \%$ were illiterate (Table 1$)$.

Table 1: Demographic characteristics of the study population

\begin{tabular}{|c|c|c|}
\hline \multicolumn{2}{|c|}{ Characteristics } & Remdesvir (N= 50) \\
\hline \multicolumn{2}{|c|}{ Age - Year } & $57.46 \pm 10.53$ \\
\hline \multirow{2}{*}{ Sex - No. (\%) } & Male & $44(88)$ \\
\cline { 2 - 3 } & Female & $6(12)$ \\
\hline \multirow{2}{*}{ Marital Status - No. (\%) } & Married & $41(82)$ \\
\hline \multirow{2}{*}{ Occupation - No. (\%) } & Widow/er & $9(18)$ \\
\hline & Business & $16(32)$ \\
\hline \multirow{2}{*}{ Socio-economic Status - No. (\%) } & Service & $15(30)$ \\
\hline & Housewife & $6(12)$ \\
\hline
\end{tabular}




\begin{tabular}{|l|c|c|}
\hline \multirow{2}{*}{ Educational Status - No. (\%) } & Upper middle class: 8-9 & $21(42)$ \\
\cline { 2 - 3 } & Upper class: 10-11 & $7(14)$ \\
\hline \multirow{3}{*}{} & Illiterate & $7(14)$ \\
\cline { 2 - 3 } & Primary & $10(20)$ \\
\cline { 2 - 3 } & SSC & $8(16)$ \\
\hline & HSC & $10(20)$ \\
\hline
\end{tabular}

Among the participants, $100 \%$ had at least one pre-existing risk factor at the time of enrolment to the study. Most prevalent co-morbidity was type 2 diabetes mellitus 34(68\%) followed by hypertension33 (66\%), bronchial asthma 07(14\%), IHD 10(20\%), dyslipidaemia 08(16\%), COPD 06(12\%), and others covered
07(14\%)(Table 2). Another important risk factor was smoking. Among the $88 \%$ male patients, 26(52\%) were current smokers. About 40(80\%) male patients had a current or previous history of smoking 14 (28\%) and $45(90 \%)$ of all patients had also passive smoking history.

Table 2: Prevalence of medical comorbidity and risk factor

\begin{tabular}{|c|c|}
\hline Medical Comorbidity and Risk Factor - No. (\%) & Remdesvir (N= 50) \\
\hline Diabetes Mellitus & $34(68)$ \\
\hline HTN & $33(66)$ \\
\hline Smoking & $26(52)$ \\
\hline IHD & $10(20)$ \\
\hline COPD & $6(12)$ \\
\hline BA & $7(14)$ \\
\hline Dyslipidaemia & $8(16)$ \\
\hline Others & $7(14)$ \\
\hline
\end{tabular}

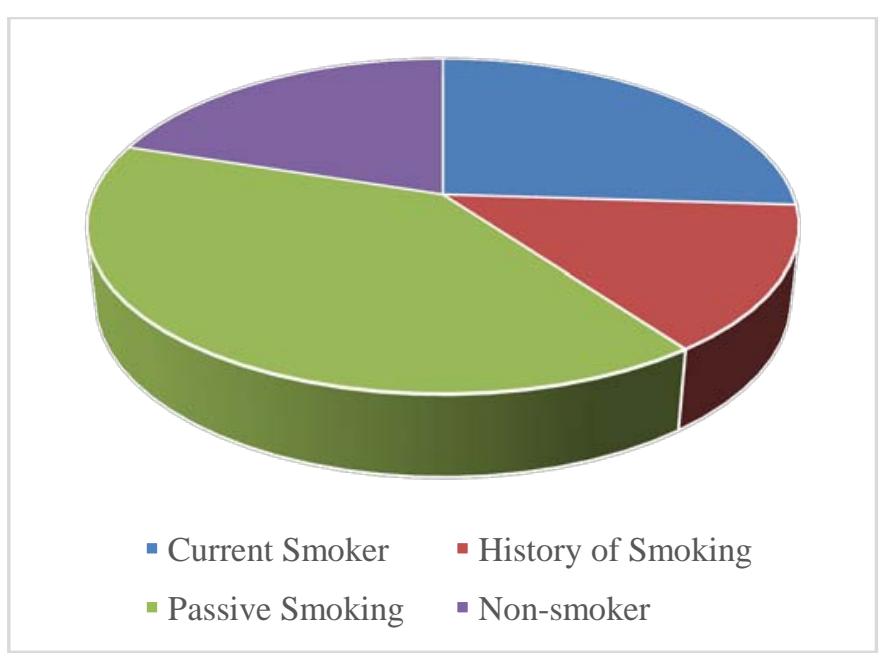

Figure 2: Status of Smoking of the Study Population

As most of the people in this country still don't utilize authorized health care facilities, most of the patients had some pre-admission treatment history including both prescribed and over the counter medications. Among prescribed medications most common was antihypertensive drugs 33(66\%), antidiabetic medications (both oral anti-diabetic drugs and insulin) 31(62\%), and lipid-lowering drugs11(22\%). Apart from the majority of the above-mentioned drugs of the patients had already consumed several over the counter medications like paracetamol 50(100\%), antibiotics 49(98\%) anti-histamine drugs 45(90\%), bronchodilators $43(86 \%)$, montelukast $42(84 \%)$, and different types of cough syrups $40(80 \%)$ (Table 3 ). 
Table 3: Pre-hospital medication consumption history

\begin{tabular}{|c|c|}
\hline Medication - No. (\%) & All (N= 50) \\
\hline Anti-hypertensive Drug & $33(66)$ \\
\hline Anti-Diabetic medications & $31(62)$ \\
\hline Lipid-Lowering drugs & $11(22)$ \\
\hline Paracetamol & $50(100)$ \\
\hline Antibiotic & $49(98)$ \\
\hline Anti-histamine drug & $45(90)$ \\
\hline Bronchodilator & $43(86)$ \\
\hline Montelukast & $42(84)$ \\
\hline Cough syrup & $40(80)$ \\
\hline
\end{tabular}

The minimum duration of symptoms was 03 days and the maximum was ten days before the admission into the hospital where the median duration of symptom onset and hospital admission was 05 days. As a presenting complaint most prevalent was shortness of breath/dyspnoea 48(96\%) and cough $46(92 \%)$ followed by fever 44(88\%), headache 30(60\%), fatigue $30(60 \%)$, vomiting $23(46 \%)$, sore throat $12(24 \%)$, loose motion 12 (24\%), confusion 12(24\%) and others
03(06\%). On physical examination, the majority of patients 47(94\%) had raised temperature(99-102.f), tachypnoea $47(94 \%)$, tachycardia 39(78\%), high blood pressure $28(56 \%)$, other significant physical findings were anaemia 18(36\%), edema 17(34\%), dehydration $06(12 \%)$, and abnormal systemic findings were mostly in respiratory system namely features of bilateral pulmonary consolidation 45(90\%), COPD 06(12\%) and unilateral consolidation 05 (10\%) (Table 4).

Table 4: Clinical characteristics of the patients at enrolment

\begin{tabular}{|l|l|c|}
\hline \multicolumn{1}{|c|}{ Duration of Symptom } & Median (days) & All (N=50) \\
\hline \multirow{5}{*}{ Symptoms - No. (\%) } & Dyspnoea & 5 \\
\hline & Cough & $48(96)$ \\
\hline & Fever & $46(92)$ \\
\hline & Headache & $44(88)$ \\
\hline & Fatigue & $30(60)$ \\
\cline { 2 - 3 } & Vomiting & $30(60)$ \\
\hline & Sore Throat & $23(46)$ \\
\hline & Loose Motion & $12(24)$ \\
\hline & Confusion & $12(24)$ \\
\hline & Others & $12(24)$ \\
\hline \multirow{5}{*}{ Signs - No. (\%) } & Raise Temperature & $3(6)$ \\
\cline { 2 - 3 } & Tachypnoea & $47(94)$ \\
\hline & No. of Patients receiving O2 at Baseline & $47(94)$ \\
\hline & Tachycardia & $50(100)$ \\
\hline & High Blood Pressure & $39(78)$ \\
\hline & Anaemia & $28(56)$ \\
\hline & Edema & $18(36)$ \\
\hline & Dehydration & $17(34)$ \\
\hline & Bilateral Pulmonary Consolidation & $6(12)$ \\
\hline & Unilateral Consolidation & $45(90)$ \\
\hline & COPD & $5(10)$ \\
\hline & & $6(12)$ \\
\hline
\end{tabular}


For the evidence of systemic involvement and as a part of routine follow-up patients had several investigations including pathological and radiological tests. The most common finding was leucocytosis $37(74 \%)$ followed by neutrophilia $36(72 \%)$, lymphopenia $35(70 \%)$, hyperglycaemia 34(68\%), raised serum creatinine $28(56 \%)$, anaemia $18(36 \%)$, and proteinuria 17(34\%). Some other important lab tests also showed supportive changes like raised ESR 50(100\%), increased CRP 50(100\%), raised serum D-dimer $45(95 \%)$, raised serum ferritin $45(90 \%)$, raised serum
LDH 43(86\%).In ECG there were some significant findings suggestive of LVH 28(56\%), IHD 10(20\%), and RVH 06(12\%). Radiology of chest also had suggestive findings like chest $x$-ray showed patchy in homogenous opacities bilaterally $49(98 \%)$ and unilaterally $01(02 \%)$ but there was also cardiomegaly in $10(20 \%)$ as well as features of COPD in 03(06\%) patients. On the other hand, HRCT of the chest showed ground-glass opacities and multiple reticulonodular shadows in $50(100 \%)$ patients in various percentages (Table 5).

Table 5: Haemato-pathological \& radiological abnormalities at baseline

Haemato-pathological \& Radiological Findings - No. (\%)

\begin{tabular}{|c|c|c|}
\hline \multirow{5}{*}{$\mathrm{CBC}$} & Raised ESR & $50(100)$ \\
\hline & Leucocytosis & $37(74)$ \\
\hline & Lymphopenia & $36(72)$ \\
\hline & Neutrophilia & $35(70)$ \\
\hline & Anemia & $18(36)$ \\
\hline RBS & Hyperglycaemia & $34(68)$ \\
\hline \multicolumn{2}{|c|}{ Raised Serum Creatinine } & $28(56)$ \\
\hline \multicolumn{2}{|c|}{ Urine R/E (Proteinuria) } & $17(34)$ \\
\hline \multicolumn{2}{|l|}{ Raised CRP } & $50(100)$ \\
\hline \multicolumn{2}{|c|}{ Raised Serum D-dimer } & $45(90)$ \\
\hline \multicolumn{2}{|c|}{ Raised Serum Ferritin } & $45(90)$ \\
\hline \multicolumn{2}{|c|}{ Raised Serum LDH } & $43(86)$ \\
\hline \multirow{3}{*}{ ECG } & LVH & $28(56)$ \\
\hline & IHD & $10(20)$ \\
\hline & $\mathrm{RVH}$ & $6(12)$ \\
\hline \multirow{4}{*}{ Chest X-Ray } & Bilateral Inhomogenus Opacity & $49(98)$ \\
\hline & Unilateral Inhomogenus Opacity & $1(2)$ \\
\hline & Cardiomegaly & $10(20)$ \\
\hline & COPD & $3(6)$ \\
\hline \multirow{2}{*}{ HRCT of Chest } & Ground Glass Opacity & $50(100)$ \\
\hline & Raticulonodular Shadow & $50(100)$ \\
\hline
\end{tabular}

a) Primary outcomes

The primary outcome was time to recovery which has been defined earlier. Treatment with Remdesivir brought an earlier recovery and patients had a median recovery time of 10 days and the average recovery time was 9.56 days. Among all the patients who received treatment during the first 07 days after the onset of symptoms had an earlier recovery than those who presented and treated later. The beneficial outcomes of Remdesivir were more when given earlier in the illness thereafter gradually reduced with the increase in the duration of symptoms. (Table 6). 
Table 6: Summary of the primary and secondary outcomes of the study population

\begin{tabular}{|c|c|c|}
\hline \multicolumn{2}{|c|}{ Outcomes } & All $(N=50)$ \\
\hline \multirow{2}{*}{ Time to Recovery - days } & Median & 10 \\
\hline & Average & 9.56 \\
\hline Mortality - No. (\%) & & $7(14)$ \\
\hline \multirow{2}{*}{ Improvement in SPO2 - \% } & By 3rd Day & 50 \\
\hline & By 7th Day & 90 \\
\hline \multirow{2}{*}{ Duration of Hospital Stay - days } & Median & 12 \\
\hline & Average & 11.46 \\
\hline Incidence of New Mechanical Ventilation - No. (\%) & & $7(14)$ \\
\hline \multirow{7}{*}{ Adverse events - No. (\%) } & AKI & $4(8)$ \\
\hline & Skin Rash & $4(8)$ \\
\hline & Jaundice & $3(6)$ \\
\hline & Nausea & $16(32)$ \\
\hline & Vomiting & $19(38)$ \\
\hline & Fatigue & $11(22)$ \\
\hline & Increased Blood Glucose & $1(2)$ \\
\hline \multirow{2}{*}{ Radiological Improvements - No. (\%) } & Chest X-Ray & $20(40)$ \\
\hline & HRCT scan of Chest & $12(24)$ \\
\hline
\end{tabular}

\section{b) Secondary outcomes}

The key secondary outcome of the study was mortality within 14 days of allocation with treatment which was $14 \%$ (07 patients). As each patient had at least one co-morbidity or risk factor, so separate analysis of the effects of pre-existing risk factor or comorbidity on mortality was not done (Table 6).

Another secondary outcome of the trial was to estimate the duration of hospital stay. The median duration of hospital stay was 12days. The maximum and minimum hospital stay was 20 days and 02 days respectively and the average duration of hospital stay was 11.46 days (Table 6 ).

All the participants were receiving oxygen at enrolment in different modes. There was a 50\% improvement in SPO2 by $3^{\text {rd }}$ day and $90 \%$ by completion of treatment with Remdesivir. For the $20(40 \%)$ patients receiving high-flow oxygen at the entry to the study, the median duration of use of this was 04 days. Among the $30(60 \%)$ patients who were not receiving non-invasive ventilation, high-flow oxygen, invasive ventilation, or ECMO at enrolment, the incidence of new noninvasive ventilation or high-flow oxygen use was $14 \%$ (07 patients). At the time of entry in the study, no patient was receiving mechanical ventilation or ECMO but during the treatment, the incidence of new mechanical ventilation was $14 \%$ (07patients), but there was no incidence of $\mathrm{ECMO}$ (Table 6).
There were various adverse events observed or reported after initiation of treatment with Remdesivir. The most common serious adverse events were acute kidney injury 08\% (04 patients), skin rash 08\% (04 patients), and jaundice 06\% (03 patients). Some other adverse events took place which was considered nonserious occurring in almost all patients included nausea (16 patients, 32\%), vomiting (19 patients, 38\%), fatigue (11 patients, 22\%), and increased blood glucose level (01 patient, 2\%)(Table 6).

Another secondary outcome was radiological improvement following treatment with Remdesivir. Among 50 patients 20 (40\%) had radiological resolutions in chest $x$-rays and $12(24 \%)$ had a resolution to the various extent in HRCT scan of the chest (Table $6)$.

\section{Discussion}

This double-blind, randomized, prospective trial showed that antiviral therapy has efficacy in the treatment of Covid-19. A rapid improvement in terms of both clinical and laboratory parameters was found after treatment with Remdesivir. A 05-day course of injectable Remdesivir reduced the hospital stay and shortened the recovery time to an average of 9.56 days and a median recovery time was 10 days. This trial also demonstrated Remdesivir effective to some extent in reducing mortality (key secondary outcome). All-cause mortality was 14\% 
in this group of severe COVID-19 patients. Moreover, Remdesivir treatment resulted in a shorter duration of hospital stay and earlier discharge from the hospital. The average hospital stay was 11.46 days with a median of 12 days.

Besides dexamethasone oxygen is the only proven supportive treatment for Coronavirus disease, so there were two secondary outcomes related to this therapy[14]. If we consider the improvement in SPO2 after initiation of treatment we find that Remdesivir may have slowed down the progression to more severe respiratory disease, as shown by the significantly rapid improvement in SPO2 following Remdesivir treatment ( $50 \%$ by $3^{\text {rd }}$ day and $90 \%$ by $5^{\text {th }}$ day), as well as a reduced incidence of new oxygen use among patients who were not receiving oxygen initially and a fewer number of patients requiring higher levels of respiratory support during the study. Besides, treatment with Remdesivir resulted in fewer days of subsequent oxygen use, and only $07(14 \%)$ patients required mechanical ventilation during the study. So, looking into all these potential benefits, this study proved that Remdesivir can play a valuable role in reducing mortality and morbidity from severe COVID-19 infections and help to materialize the plan of the judicial use of limited health care resources.

The findings in our study should have a comparison in similar outcomes with those observed in other randomized trials of Remdesivir. The first stage of the Adaptive Covid-19 Treatment Trial (ACTT-1) funded by the National Institute of Allergy and Infectious Diseases and others; randomized a total of 1062 patients (with 541 assigned to Remdesivir and 521 to placebo). Those who received Remdesivir had a median recovery time of ten days as compared with 15 days among those who received placebo. This study also demonstrated that the patients who received Remdesivir were found to be more likely than those who received placebo to have clinical improvement at day 15 . The Kaplan-Meier estimates of mortality were $6.7 \%$ with Remdesivir and $11.9 \%$ with placebo by day 15 . Serious adverse events were reported in 131 of the 532 patients who received Remdesivir (24.6\%) and in 163 of the 516 patients who received placebo (31.6\%)[15]. Most of the findings of this large scale study have a proximity to the outcomes of our study except the mortality rate. But in this study, there were mild, moderate, and severe cases where we included only severe cases. So, high mortality in respect to that study is quite inevitable.

Early in the pandemic Wang et al. enrolled 237 patients (158 assigned to Remdesivir and 79 to placebo) in China and found a shorter time to improvement (a two-point improvement) with Remdesivir: 21.0 days $(95 \% \mathrm{Cl}, 13.0$ to 28.0$)$ in the Remdesivir group and 23.0 days (95\% Cl, 15.0 to 28.0) in the placebo group[16]. But that trial did not complete full enrolment owing to local control of the outbreak.

In another open-label, randomized study of remdesivir in hospitalized patients with moderateseverity Covid-19 (83\% were not receiving oxygen at baseline),those randomized to a 10-day course of remdesivir did not have a statistically significant difference in clinical status compared with standard care at 11 days after initiation of treatment. Patients randomized to a 5-day course of remdesivir had a statistically significant difference in clinical status compared with standard care (odds ratio, $1.65 ; 95 \% \mathrm{Cl}$, 1.09 to $2.48 ; P=0.02)[17]$.

It was a tough task to accomplish the trial during an unpredictable and sudden outbreak of a pandemic. There was not only a loss of lives but also a massive economic shutdown. The research team was simultaneously carrying out their hospital duties alongside conducting this trial. Three trial sites were placed in different places. Moreover, there was a scarcity of medications, personal protective equipment, sample taking facilities and trial-related supplies, investigation facilities, and an also different set of workers with shifting and roster duties which brought a lack of fascination to complete the study. However, our research team overcame all these obstacles and hardships with vigorous physical and intellectual efforts. As a result, we were able to enroll in a diverse population, similar to the population that was being infected with SARSCoV-2 during this pandemic.

\section{Limitations}

Despite the tremendous effort of an extraordinarily co-operative team, the study lagged in several aspects. Firstly, it was tough to allot a large population in this treatment arm due to rapidly evolving national and international treatment protocols. As a result, the sample size could not be big enough to make any strong interpretation. Secondly, all three trial sites were distant from each other having individual authorities and working stuff. So, to maintain a uniform treatment protocol everywhere was not possible in each case. Thirdly, in this trial, we only enrolled severe disease patients. This resulted in high mortality rates in comparison with other similar studies and it was difficult to make any comment on the efficacy of Remdesivir in other spectrums of the disease. Finally, as we only monitored the patients upto 14 days or their discharge from the hospital, we could not evaluate any late complications related either to the drug or disease itself.

\section{Conclusion}

The COVID-19 pandemic is still going on and there are catastrophic consequences not only in the health sector but also massive socio-economic collapse around the world. It seems that this pandemic is 
unstoppable and the search for an effective drug or vaccine is also never-ending. Considering all the facts and realities it can be said that despite several limitations this study can guide us in several ways. The results obtained from this trial can be used as preliminary data to design a more large scale study. This study can be a milestone in the way to find out a fruitful agent to fight against the COVID-19 pandemic.

\section{References Références Referencias}

1. S. Perlman, "Another Decade, Another Coronavirus," N. Engl. J. Med., vol. 382, no. 8, pp. 760-762, Feb. 2020, doi: 10.1056/nejme2001126.

2. Y. A. Helmy, M. Fawzy, A. Elaswad, A. Sobieh, S. P. Kenney, and A. A. Shehata, "Clinical Medicine The COVID-19 Pandemic: A Comprehensive Review of Taxonomy, Genetics, Epidemiology, Diagnosis, Treatment, and Control," vol. 9, no. 4, p. 1225, 2020, doi: 10.3390/jcm9041225.

3. M. Chan-Yeung and R.-H. XU, "SARS: epidemiology," Respirology, vol. 8, no. s1, pp. S9S14, Nov. 2003, doi: 10.1046/j.1440-1843.2003. 00518.x

4. S. Joshi et al., "Role of favipiravir in the treatment of COVID-19," Int. J. Infect. Dis., Oct. 2020, doi: 10.1016/j.jijid.2020.10.069.

5. A. PG, "Coronavirus COVID-19 (SARS-CoV-2)," vol. 19, pp. 1-17, 2020.

6. V. C. Yan and F. L. Muller, "Gilead should ditch remdesivir and focus on its simpler ancestor STAT," 2020. [Online]. Available: https://www.stat news.com/2020/05/14/gilead-should-ditch-remdesivir-and-focus-on-its-simpler-safer-ancestor/.

[Accessed: 16-Dec-2020].

7. T. P. Sheahan et al., "Comparative therapeutic efficacy of remdesivir and combination lopinavir, ritonavir, and interferon beta against MERS-CoV," Nat. Commun., vol. 11, no. 1, 2020, doi: 10.1038/ s41467-019-13940-6.

8. M. L. Agostini et al., "Coronavirus susceptibility to the antiviral remdesivir (GS-5734) is mediated by the viral polymerase and the proofreading exoribonuclease," MBio, vol. 9, no. 2, pp. 1-15, 2018, doi: 10.1128/mBio.00221-18.

9. M. Wang et al., "Remdesivir and chloroquine effectively inhibit the recently emerged novel coronavirus (2019-nCoV) in vitro," Cell Res., vol. 30, no. 3, pp. 269-271, 2020, doi: 10.1038/s41422-0200282-0.

10. E. de Wit et al., "Prophylactic and therapeutic remdesivir (GS-5734) treatment in the rhesus macaque model of MERS-CoV infection," Proc. Natl. Acad. Sci. U. S. A., vol. 117, no. 12, pp. 6771-6776, 2020, doi: 10.1073/pnas.1922083117.

11. WHO, "Therapeutics and COVID-19," no. November, 2020.
12. F. Lamontagne et al., "A living WHO guideline on drugs for covid-19," BMJ, vol. 370, Sep. 2020, doi: 10.1136/bmj.m3379.

13. DGHS, "National Guidelines on Clinical Management of COVID-19," vol. 7, no. November, May 2020.

14. The Recovery Collaborative Group, "Dexamethasone in Hospitalized Patients with Covid-19 Preliminary Report | Enhanced Reader," 2020. [Online]. Available: chrome-extension: //dagcmkpag jlhakfdhnbomgmjdpkdklff/enhanced-reader.html? openApp\&pdf=https\%3A\%2F\%2Fwww.nejm.org\% 2Fdoi\%2Fpdf\%2F10.1056\%2FNEJMoa2021436\%3F articleTools\%3Dtrue. [Accessed: 16-Dec-2020].

15. J. H. Beigel et al., "Remdesivir for the Treatment of Covid-19 - Final Report," N. Engl. J. Med., vol. 383, no. 19, pp. 1813-1826, 2020, doi: 10.1056/ nejmoa2007764.

16. Y. Wang et al., "Remdesivir in adults with severe COVID-19: a randomised, double-blind, placebocontrolled, multicentre trial," www.thelancet.com, vol. 395, p. 2020, 2020, doi: 10.1016/S01406736(20)31022-9.

17. C. D. Spinner et al., "Effect of Remdesivir vs Standard Care on Clinical Status at 11 Days in Patients with Moderate COVID-19: A Randomized Clinical Trial," JAMA - J. Am. Med. Assoc., vol. 324, no. 11, pp. 1048-1057, 2020, doi: 10.1001/jama. 2020.16349 . 